\title{
Erratum to: Impact of FTO genotypes on BMI and weight in polycystic ovary syndrome: a systematic review and meta-analysis
}

\author{
P. Wojciechowski - A. Lipowska • P. Rys • K. G. Ewens • S. Franks • S. Tan • \\ E. Lerchbaum • J. Vcelak • R. Attaoua - M. Straczkowski • R. Azziz • T. M. Barber • \\ A. Hinney • B. Obermayer-Pietsch • P. Lukasova • B. Bendlova • F. Grigorescu • \\ I. Kowalska • M. O. Goodarzi • GIANT Consortium • J. F. Strauss III • \\ M. I. McCarthy • M. T. Malecki
}

Published online: 27 July 2012

(C) Springer-Verlag 2012

\section{Erratum to: Diabetologia \\ DOI 10.1007/s00125-012-2638-6}

Unfortunately there was an error in the contribution statement for this paper. The correct version is given here, with the amendment shown in colour.

PW and PR developed the protocol; PW, AL, ST, PL searched the medical databases and carried out data analysis.

The online version of the original article can be found at http://dx.doi.org/ 10.1007/s00125-012-2638-6.

\footnotetext{
P. Wojciechowski $\cdot$ P. Rys

HTA Consulting,

Krakow, Poland
}

A. Lipowska

School of Medicine, Emory University,

Atlanta, GA, USA

\section{K. G. Ewens}

Department of Genetics, School of Medicine,

University of Pennsylvania,

Philadelphia, PA, USA

\section{S. Franks}

Institute of Reproductive and Developmental Biology,

Imperial College London, Hammersmith Hospital,

London, UK

\section{S. Tan}

Department of Endocrinology, University of Duisburg-Essen,

Essen, Germany

E. Lerchbaum $\cdot$ B. Obermayer-Pietsch

Department of Internal Medicine, Division of Endocrinology and Metabolism, Medical University of Graz,

Graz, Austria
PW, PR, KGE, SF, ST, EL, JV, RAt, MS, RAz, TMB, AH, BOP, PL, BB, FG, IK, MOG, JFSIII, MMcC, MTM researched and interpreted data; PW, AL, PR, KGE, IK, JFSIII, MTM wrote the manuscript. KGE, SF, ST, EL, JV, RAt, MS, RAz, TMB, AH, BO-P, BB, FG, MOG, JFSIII, MMcC, PL critically reviewed the manuscript; MTM designed the study and coordinated the project. All authors gave final approval of the version of the manuscript to be published.

J. Vcelak $\cdot$ P. Lukasova $\cdot$ B. Bendlova

Institute of Endocrinology,

Prague, Czech Republic

R. Attaoua $\cdot$ F. Grigorescu

Molecular Endocrinology Laboratory, UMR-204 NUTRIPASS, Institut Universitaire de Recherche Clinique (IURC),

Montpellier, France

M. Straczkowski $\cdot$ I. Kowalska

Department of Endocrinology, Diabetology and Internal Medicine, Medical University of Bialystok,

Bialystok, Poland

\section{R. Azziz}

Department of Obstetrics and Gynecology,

Georgia Health Sciences University,

Augusta, GA, USA

\section{T. M. Barber}

Department of Metabolic and Vascular Health, Clinical Sciences Research Laboratories, Warwick Medical School,

Coventry, UK 


\footnotetext{
A. Hinney

Department of Child and Adolescent Psychiatry,

University of Duisburg-Essen,

Essen, Germany

M. O. Goodarzi

Division of Endocrinology, Diabetes, and Metabolism,

Cedars-Sinai Medical Center,
}

Los Angeles, CA, USA

J. F. Strauss III

Department of Obstetrics and Gynecology,

Virginia Commonwealth University,

Richmond, VA, USA

M. I. McCarthy

Wellcome Trust Centre for Human Genetics, University of Oxford,

Oxford, UK

M. I. McCarthy

Oxford Centre for Diabetes, Endocrinology and Metabolism,

University of Oxford,

Oxford, UK

M. T. Malecki $(\bowtie)$

Department of Metabolic Diseases,

Jagiellonian University Medical College,

15 Kopernika Street,

31-501, Krakow, Poland

e-mail: malecki_malecki@yahoo.com

M. T. Malecki

University Hospital,

Krakow, Poland 